\title{
"iAcá no hay maricones!" Experiencia de lectura y escritura en el Centro Cerrado Almafuerte de la ciudad de La Plata
}

\author{
"There aren't fags here!" Experience of reading and writing at a Center of \\ Confinement in the city of La Plata
}

\author{
Luciana Daniela Morini \\ lucyanamorini@gmail.com \\ CONICET - Facultad de Humanidades y Ciencias \\ de la Educación. Universidad Nacional de La Plata, \\ Argentina
}

Recepción: 01 Junio 2019

Aprobación: 01 Marzo 2021

Publicación: 01 Septiembre 2021

Cita sugerida: Morini, L. D. (2021). “ ¡Acá no hay maricones!” Experiencia de lectura y escritura en el Centro Cerrado Almafuerte de la ciudad de La Plata. Descentrada, 5(2), e151. https://doi.org/10.24215/25457284e151
Resumen: El presente trabajo analiza una situación de enseñanza-aprendizaje en una clase de Literatura, en un curso que aúna estudiantes de quinto y sexto año de la Escuela Secundaria Nro. 40, que articula dentro del Centro Cerrado Almafuerte en la localidad de La Plata, el cual aloja a varones jóvenes en conflicto con la ley penal.

A partir del análisis de un registro de una clase en la cual se leyó el cuento "El marica" de Abelardo Castillo, se desprenden múltiples interrogantes en torno a las categorías sexo-genéricas y a los roles masculinos y femeninos históricamente construidos. A su vez, desde un enfoque etnográfico con perspectiva de género, se busca reflexionar sobre el espacio institucional, las corporalidades, el uso del lenguaje, las formas de la resistencia a través de la parodia, las normas de control corporal y subjetivo, y el vínculo entre la docente y los estudiantes protagonistas de la experiencia. Por último, se señala el potencial de la literatura para desarrollar debates en torno a estas temáticas.

Palabras clave: Educación, Sexualidad, Contexto de encierro, Literatura.

Abstract: The present work analyzes a teaching-learning situation in a literature class in a course that brings together fifth and sixth year students of the Secondary School No. 40 that articulates within the Almafuerte Confinement Center in the city of La Plata, which houses young men in conflict with criminal law.

From the analysis of a class register in which Abelardo Castillo's story "The Faggot" was read, multiple questions arise regarding sex-gender categories and historically constructed male and female roles. At the same time, from an ethnographic approach with a gender perspective, we seek to reflect on the institutional space, the corporeality, the use of language, the forms of resistance through parody, the norms of corporal and subjective control and the link between the teacher and the students who are the protagonists of the experience. Finally, it points out the potential of literature to develop debates around these themes.

Keywords: Education, Sexuality, Context of confinement, Literature. 


\section{INTRODUCCIÓN}

El presente artículo tiene como objetivo problematizar una situación de enseñanza que se generó a partir de la lectura del cuento "El Marica" de Abelardo Castillo en una clase de Literatura, que tuvo lugar en un Centro Cerrado de jóvenes varones.

Desde un enfoque etnográfico con perspectiva de género, a partir del análisis de un registro áulico se desprenden múltiples interrogantes, principalmente en torno a las categorías sexo-genéricas y a los roles masculinos y femeninos históricamente construidos. Asimismo, se destaca el potencial de la literatura para propiciar debates en torno a estas temáticas.

$\mathrm{Al}$ mismo tiempo, también se abordan reflexiones sobre el espacio institucional, las corporalidades, el uso del lenguaje, las formas de la resistencia a través de la parodia, las normas de control corporal y subjetivo, y el vínculo entre docente y estudiantes. Por ello, lo consideramos un disparador para pensar estas múltiples discusiones y tensiones a partir de una experiencia concreta.

\section{MARCo TEÓRico Y Metodología}

El presente artículo parte de una experiencia docente con jóvenes en contextos de encierro, privados eventualmente de su libertad en el Centro Cerrado Almafuerte, en la ciudad de La Plata. Los Centros Cerrados son establecimientos de régimen cerrado, para el cumplimiento de medidas privativas de la libertad ordenadas por la Justicia en el marco de un proceso penal y destinados a alojar a población masculina y/o femenina hasta 18 años de edad. Esta experiencia específica tuvo lugar en la Escuela Secundaria Nro. 40, que articula dentro de la misma institución en la que permanecen los jóvenes, durante una clase de Literatura, en un curso que aúna estudiantes de quinto y sexto año. Cabe destacar que, al momento de la presente experiencia áulica, solo cursaban la asignatura cuatro estudiantes, ya que la mayoría de los jóvenes que allí residían, se encontraban cursando los primeros años de la escuela secundaria.

Durante un período sostenido en el tiempo, aproximadamente un ciclo lectivo completo, se registró el cotidiano escolar al interior de las asignaturas Literatura y Prácticas del Lenguaje. Este análisis se desprende de uno de los registros de clase, elaborado dentro de un trabajo de campo más amplio, cuya investigación tiene como objeto de estudio el análisis de las prácticas de lectura, escritura y oralidad que se desarrollan dentro de los Centros Cerrados Almafuerte, en la ciudad de La Plata y Eva Perón, en la localidad de Abasto, perteneciente al partido de La Plata.

El trabajo se centra en el análisis de una clase en la cual se leyó en voz alta y en conjunto el cuento "El marica" (2007 [1961]) de Abelardo Castillo. A partir de las discusiones que tuvieron lugar en el espacio áulico, del análisis del registro de la presente clase y de las consignas de escritura propuestas por la docente y realizadas por los estudiantes, se intentará dar cuenta de qué ideas, valores y sentidos en torno a los conceptos de género, identidades y sexualidades tienen los estudiantes pertenecientes a dicha institución.

Entendemos que los Centros Cerrados de menores varones -como así también otros contextos de encierro como centros de rehabilitación de adicciones y unidades penitenciarias, entre otros- poseen una dinámica particular. En principio, son espacios donde prima lo masculino, fundamentalmente por las personas que lo habitan, ya que los varones son quienes permanecen la mayor parte del tiempo (jóvenes varones reclusos, guardiacárceles, preceptores, directores) y algunos cumplen funciones de control y administración; mientras que las mujeres ingresan y egresan de este espacio con menor regularidad, suelen ser maestras, profesoras, psicólogas y trabajadoras sociales, quienes poseen otros roles, históricamente asociados a la contención y el cuidado. A su vez, la idea de masculinidad gira en torno a las distintas prácticas que tienen lugar y a los comportamientos esperados, los cuales se rigen bajo parámetros en relación con lo viril, la fuerza física, el poder, la resistencia y la valentía, entre otros. En este sentido, siguiendo a Oleastro (2018) podemos pensar 
en ciertas prácticas para sobrevivir al encierro, como la construcción diaria del "respeto" (Bourgois, 2010) a ser ganado y el "aguante" sostenido en diferentes actos. Retomaremos estas ideas más adelante.

Este análisis se enmarca en una perspectiva etnográfica ya que consideramos que este estilo de investigación permite estudiar procesos educativos que poseen cierta complejidad, la cual se busca comprender y eventualmente desandar para generar aportes significativos a la construcción de conocimiento. Como señala Elsie Rockwell, a partir de un trabajo de campo sostenido en el tiempo "lo que el etnógrafo hace es documentar lo no-documentado de la realidad social, (...) en las sociedades modernas lo no-documentado es lo familiar, lo cotidiano, lo oculto, lo inconsciente" (2009, p. 21).

La metodología que se ha utilizado es la observación participante ya que se han desarrollado tareas docentes en el espacio donde se llevó adelante la presente práctica de lectura y escritura, objeto de estudio de nuestro análisis. De esta manera, se buscará poner en relación el espacio en el que dichas prácticas tienen lugar, las personas protagonistas, así como las escrituras de los estudiantes a modo de resolución de consignas.

\section{El Centro Cerrado Almafuerte}

El Centro Cerrado Almafuerte se encuentra ubicado en el barrio Melchor Romero, sobre la Avenida 520 entre las calles 182 y 183 .

Los estudiantes de dicha institución conforman un rango etario que va de los 15 a los 18 años. En su mayoría, provienen de diversos barrios periféricos y villas de emergencia de diferentes localidades de la provincia de Buenos Aires, principalmente del conurbano. La diversidad de lugares de procedencia y de niveles de escolaridad alcanzados -principalmente en los cursos de "pluriaño" que aúnan estudiantes de primero, segundo y tercer año- conlleva a que sea el o la docente quien decida qué metodología empleará al interior del espacio áulico.

Tal es así, que para el curso en el que se ancla el presente análisis, el cual engloba estudiantes de quinto y sexto año, como decisión metodológica se optó por seleccionar ciertos contenidos y textos literarios de ambos Diseños Curriculares de Literatura para la Educación Secundaria de la Provincia de Buenos Aires y trabajar en conjunto. El cuento "El marica" se leyó en clase junto con otros relatos argentinos breves que podrían englobarse bajo la categoría de "realistas", contenido de sexto año. Al mismo tiempo, se buscó focalizar en un narrador en segunda persona.

El cuento, publicado en 1961 en Las otras puertas, narra, a través de la voz de Abelardo, la iniciación sexual de un grupo de jóvenes con "la gorda”, una prostituta del pueblo. El relato está ubicado en un tiempo posterior donde Abelardo le "habla” a César, "el marica”, rememorando la situación. Cabe señalar que ni Abelardo ni César concretaron el acto sexual esa noche.

\section{Registro de Clase}

A continuación, se transcribirá el registro de la clase. Luego, algunas respuestas de los jóvenes a las consignas solicitadas por la docente.

Los estudiantes son requisados ${ }^{1}$ en una habitación aledaña al aula por los guardiacárceles -llamados maestros en el interior de la institución- y luego ingresan a ésta. Una vez que están todos adentro, entra la docente. Es el cumpleaños de Facundo, ${ }^{2}$ la docente le regala un chocolate. Facundo está parado en una esquina. Mauro y Bruno están sentados en el banco. Coco está serio y no habla. Después de conversar sobre cosas ajenas a la materia, como las visitas del fin de semana y los saludos por el cumpleaños, entre otros temas, la docente reparte las fotocopias del cuento "El marica" y pregunta si alguien quiere leer en voz alta.

Hacia la mitad de la primera carilla, Facundo empieza a introducir otras palabras mientras lee que causan gracia entre sus compañeros, pero no por ello interrumpe la lectura. En un momento la docente le pide que 
pare con la broma porque Coco y Bruno no están siguiendo la lectura con la vista, sino que la escuchan y se van a confundir. Mauro le responde a la docente, argumentando que no se confunden. Facundo continúa con la lectura.

Cabe destacar algunos de los cambios introducidos por Facundo: en primer lugar, el personaje de "la gorda" fue modificado por "la papuda", luego pasó a ser "la gordi" y, una vez finalizada la lectura, los estudiantes le contaron a la docente que relacionaban al personaje con una trabajadora social que iba antes al Centro, la cual era gorda y los jóvenes se cargaban con ella unos a los otros. Finalmente, al momento de escribir los personajes en el pizarrón la llamaron "diegor". Por otro lado, cuando aparece el personaje "el negro" Facundo lee "yo" porque le dicen así por su tono de piel. Por ejemplo: Verle la cara a Dios, dije yo. Más adelante, Facundo se retracta y argumenta que él no quiere estar casado con la gorda, a lo que vuelve a decir "el negro" como en el texto original. Por último, los personajes que van junto con Abelardo y César a la casa de la gorda con el fin de debutar sexualmente, se llaman Roberto, Cacho y el colorado, pero Facundo nombra a Titi, El flaco y Pablo, tres estudiantes de primer año a quienes la docente conoce ya que tiene clases de Prácticas del Lenguaje con ellos los días miércoles.

En el momento en que nombra a estos otros compañeros, Mauro le llama la atención, diciéndole que no sea zarpado, que afloje con los berretines. ${ }^{3} \mathrm{~A}$ su vez, cuando Bruno fuma cerca de la ventana, Mauro también le llama la atención, argumentando que "se regala solo" fumando ahí, que no sea gil que los van a engomar ${ }^{4}$ a todos.

Una vez finalizada la lectura, se hacen algunos comentarios sobre el cuento y luego la docente copia una guía de lectura en el pizarrón. Uno de los ítems proponía el siguiente ejercicio de producción de escritura:

"Escribir una carta en primera persona (como si fueras César) donde le respondas a Abelardo. ¿Qué le dirías después de varios años?”

Esta consigna dio lugar al siguiente incidente:

Coco: Conmigo no cuentes

Bruno: Conmigo tampoco

Profesora: Pero ¿por qué?

B: Porque vos querés que digamos que somos putos. Está re loca esta.

P: No, no. ¡no que ustedes son putos! Cuando un autor escribe algo, con voz de alguien, está creando un personaje. No es el autor.

F: Sí, pero de ahí a escribir como puto... (risas)

P: Pero es un ejercicio. Además, la respuesta puede ser otra cosa... que no lo son, justamente. En ningún momento se dice que César lo era. Queda en la interpretación de cada lector.

F: ¿Y qué querés que pongamos? "Eh huacho estas re zarpado en puto" (risas)

C: Estamos en la cárcel profe, queda mal eso.

M: Mirá si nos la agarra alguien.

F: Olvídate

P: ¿Y si la hacen acá y me la dan? No la va a ver nadie, la hacen en clase y me la llevo.

B: No.

P: Bueno, esta vez ganan ustedes. Pero la próxima consigna de escritura la hacen.

C: El otro día, que escribamos sobre los chivos... Ahora sobre los putos...

P: ¿Ninguno tiene un amigo gay?

M: Sí, él. (Abraza a Facundo a modo de broma)

B: No... ni a palos... acá es boleta.

P: ¿Y afuera?

M: Tampoco

B: ¿Usted sí?

P: Sí, varios. Y amigas lesbianas también (...)

P: Bueno, volvamos al texto ¿qué les parece que está dando a entender el final del texto, de manera implícita?

F: Que no pudo verle la cara a Dios

M: Verle la cara a Dios (risas) ${ }^{5}$

P: Claro... pero ¿por qué? B: Porque se fue corriendo. 


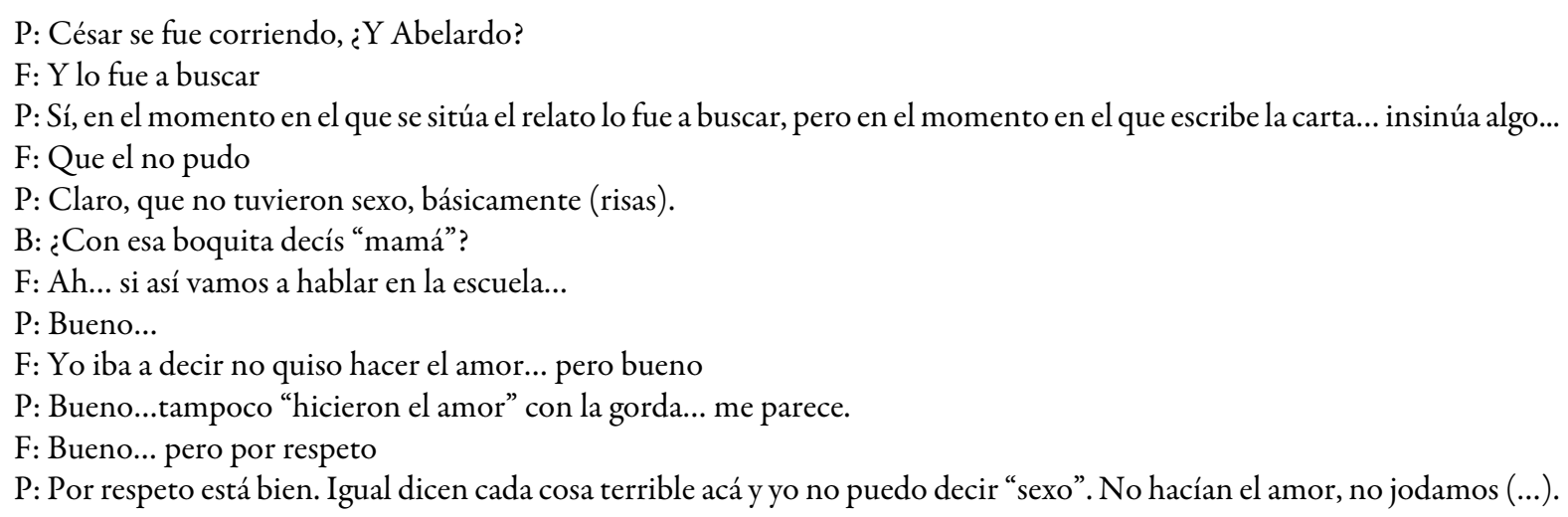

Algunas respuestas de los cuestionarios fueron las siguientes: ${ }^{6}$

"¿Qué pensás respecto de la actitud del narrador para con su amigo?"

-Pienso que estuvieron mal los dos porque ninguno concretó lo que tenía que concretar (Mauro)

- Al respecto de la actitud para con César, pienso que estuvieron sarpado porque uno lo obligaba y el otro porque no se la "garchó"(Coco)

- Pienso es un homosexual reprimido o arrepentido porque no se animó a decirle lo que sentía (Facundo)

"¿Por qué el narrador dice que César es raro?"

- Porque no se subia a los bondis ${ }^{7}$ que hacían los otros (Bruno)

- El narrador dice que Cesar es raro porque no hacia lo mismo que otros pibes. Por ejemplo no meaba delante de los otros. (Coco)

- No voy a opinar sobre esta pregunta, por que a mi me gustan las mujeres. (Facundo)

"¿Qué puede estar insinuando el final del cuento? ¿Qué da a entender de manera implícita?"

- No pega una con su amigo y aparentemente el mismo también es gay (Bruno)

- El final del cuento insinua que los dos eran homosexual y ninguno se garcho a la gordi y que se gustaban. (Coco)

- Que Avelardo tampoco le gustaban las mujeres y el tampoco pudo con la gorda. En la forma da a entender que Avelardo era igual que César. (Facundo)

"¿Por qué crees que años más tarde decide escribirle/llamarlo?"

- El quedo bien por que decia que avia estado con la gorda pero no estuvo y el otro quedo re mal por que no estuvo (Mauro)

“:Por qué crees que el autor elige un homónimo, es decir, llamar al personaje igual que su propio nombre: Abelardo?”

- Por que no quiere que todas las personas sepan su sexualidad (Facundo)

\section{ANÁLISIS}

A partir del análisis del registro de clase y de las respuestas de los estudiantes se desprenden diferentes cuestiones a analizar. En primer lugar, puede analizarse cómo se posicionan los cuerpos en el espacio áulico, a diferencia, tal vez, de los otros espacios que habitan dentro de esta institución total (Goffman, 2009) ${ }^{8}$. Los cuerpos son continuamente disciplinados, sometidos a distintas rutinas diarias que se repiten innumerables veces por día, como la requisa. Los jóvenes-internos tienen horarios fijos para despertarse, desayunar, asistir a la escuela, realizar las tareas encomendadas (muchos están encargados de actividades como la limpieza o fajina, al igual que en unidades penitenciarias de adultos). La administración del espacio físico, del tiempo, de las condiciones habitacionales, la comunicación, la visita, la alimentación entre otros, están regulados con el objetivo de mantener el orden interno y disciplinar a los individuos. Abonan así a diversos "procedimientos de mortificación del yo" (Goffman, 2009). Otros autores también han puesto el foco en estos múltiples 
procesos de degradación del yo, consecuencia del aparato disciplinador, como Bergalli (1980); Cesaroni (2010); García Borés (2003); Valverde (1997), por solo nombrar algunos.

Volviendo a nuestro caso de estudio, en la institución se censuran gestos, volúmenes de voz, determinadas vestimentas y adornos (se les prohíbe la utilización de piercings, por ejemplo), inclusive la palabra en determinadas situaciones.

$\mathrm{Al}$ ingresar al espacio áulico estas corporalidades están presentes. Los jóvenes se desparraman sobre el banco, se paran, se golpean, se tocan. Se rompe toda quietud y todo silencio. Los estudiantes participan de la clase permaneciendo parados junto a la ventana, sentados sobre el banco, fumando o tomando mate. El ambiente, con esos cuerpos en movimiento y no sentados mirando hacia el pizarrón, de forma homogénea, hacia el lugar donde se posiciona la docente, podría resultar disruptivo si pensamos en que históricamente se ha instalado la idea de que para que se desarrolle una situación de enseñanza-aprendizaje "exitosa" los cuerpos deben estar posicionados de determinada manera hacia el interior del aula. De esta manera, creemos que el espacio escolar, áulico, se perfila como un espacio "otro", distinto al pabellón y a la rutina cotidiana, que permite, habilita y propone otros movimientos, otras discusiones y perspectivas.

En segundo lugar, nos interesa resaltar lo que hace a la dimensión de la lengua y cómo esta puede relacionarse con problemáticas sexo-genéricas. En principio, advertimos palabras propias de un léxico compartido por los estudiantes: bondi, berretines, zarpado, garchar, entre otras. Respecto al habla de los jóvenes de barrios populares, y la utilización de los "berretines”, Rodríguez Alzueta y Garibaldi Noya (2016) en el libro Hacer bardo, provocaciones, resistencias y derivas de jóvenes urbanos dicen que "los berretines son contraseñas de acceso o tránsito a los grupos de pares (...) el berretín es la palabra tallada al interior de la grupalidad (...) es la palabra más el silencio. Hablan para los entendidos, para el resto es como si no hablaran. Visto desde afuera los pibes, con los berretines, se vuelven ininteligibles" (p. 142). En el registro la utilización excesiva de berretines por parte de Facundo es acallada por Mauro. En el libro se lee: "No hay que abusar de los berretines, sobre todo al interior del grupo. Hay que nombrarlos de vez en cuando para entrenarlos, pero conviene no alardear con ellos" (p. 143). A su vez, el estudio citado informa que los institutos de menores, en tanto espacios cerrados y cifrados, se perfilan como "el mejor lugar para entrenarlos" (p.143). Los berretines son, en palabras de los autores, santo y seña (p.142), tanto en el interior del grupo de pares, como en el momento de encontrarse o enfrentarse con otros grupos. Así, en una de las respuestas de Bruno leemos "Por que no se subia a los bondis que hacian los otros". Bondi, en este caso, significa conflicto, problema, lío, quilombo.

Por otro lado, observamos que en el momento en que utiliza la palabra "sexo" la docente es ridiculizada, juzgada por los estudiantes, mientras que uno de ellos resuelve la consigna dejando por escrito la palabra garchar. Esto genera la impresión de que, para los estudiantes, lxs docentes no deben decir esas palabras, sino hablar con una cierta propiedad, dejando las "malas palabras" y los berretines, para el estudiantado y la juventud en general. Se cree que esta concepción es reforzada por el hecho de que la docente es mujer y por tanto responde a la representación histórica de la mujer-maestra como "segunda madre".

Entonces, a partir de pensar este espacio como un lugar donde prima lo masculino y donde las mujeres que circulan por la institución (profesoras, maestras, psicólogas, trabajadoras sociales y, también, la "visita" de madres, hermanas y novias), son asociadas a roles de educación, contención y cuidado del otro, se desprende la posibilidad de problematizar la idea que desarrolla Lagarde respecto de las mujeres como "seres para otros" (2001). Lagarde desarrolla una mirada histórico-política sobre el amor, intentando desandar esa exigencia impuesta para las mujeres. Señala han sido educadas para el amor, cuya razón de ser emana de la existencia de los demás y no de la propia.

Asimismo, el registro áulico posibilita plantear interrogantes respecto de la condición de ser mujer y docente, principalmente sobre qué se espera de ella, en este caso, a partir de ciertos juicios de valor emitidos por los estudiantes con respecto al lenguaje utilizado. Interrogantes y problemáticas que exceden los fines del presente trabajo, pero que juzgamos interesante profundizar y destacar. En este sentido, creemos que 
dos obras claves que abordan la temática de género y docencia, podrían iluminar el asunto. En primer lugar, Morgade (1997) en Mujeres en la educación. Género y docencia en la Argentina 1870-1930, sostiene que el trabajo docente reproduce los modelos estereotipados de lo femenino y lo masculino. En el libro, compila una serie de artículos cuya premisa principal es que las mujeres llegaron a ser docentes "naturalmente" (Morgade, 1997, p.13) como han llegado a ser madres. Así, da cuenta de diversas condiciones sociales, históricas, políticas y económicas que tuercen la idea de "naturalidad". En segundo lugar, Yannoulas (1994) en Educar: ¿una profesión de mujeres? La feminización del normalismo y la docencia (1870-1930) analiza el proceso de feminización de la docencia en Argentina y Brasil. A partir del análisis de la división sexual del trabajo, sostiene que esta división selecciona distintos ámbitos en los cuales han de desenvolverse las personas, de modo tal que las mujeres se desarrollan dentro del ámbito privado y los hombres dentro del ámbito público. De esta manera, distintas profesiones responden a disímiles valores sociales, hecho que genera que la docencia sea una profesión que se ha feminizado por estar asociada a cualidades que tienen que ver con lo pasivo, lo comprensivo y lo paciente.

Por otra parte, retomando la dimensión del contexto: un espacio asociado a la idea de "masculinidad" -en tanto un objeto histórico y cultural que se inscribe en un régimen de género (Connell, 1987)- y de "heterosexualidad obligatoria" (Rubin, 1986), podemos relacionar la percepción que los jóvenes poseen de la institución en la cual se encuentran alojados con la negación de los cuatro estudiantes a resolver la consigna de producción de escritura. Advertimos que en un principio existe una negación rotunda a realizar la consigna ante la posibilidad de que su masculinidad se encuentre amenazada. Pero el problema no es la consigna en sí, si no el "qué dirán". Tampoco ensayan posturas hacia el interior del aula, sino que el problema, la amenaza, la constituye el afuera del aula: los otros jóvenes con quienes se comparten otros espacios y que, por no asistir a esa clase, no entenderían la razón de ser de esa escritura, por lo tanto, si esos escritos son leídos por otros jóvenes, podrían dar lugar a pensar que son sus voces, sus individualidades las que hablan. En este sentido, retomamos a Oleastro (2018) quien a propósito de un estudio sobre el cuerpo y la sexualidad en cárceles de varones sostiene que:

Las relaciones son dinámicas, pero a la vez están estructuradas por códigos que operan en las acciones de los detenidos y que permiten posicionarse frente a los demás. Esto genera una imagen de sí mismo; no sólo importa lo que se hace sino cómo se hace y cómo se muestra eso frente al resto. (Oleastro, 2018, p. 70).

$\mathrm{Al}$ mismo tiempo, si observamos una de las respuestas: No voy a opinar sobre esta pregunta, por que ami me gustan las mujeres, podemos pensar en ciertos micro-machismos (Bonino, 2004), que se ejecutan en función de las exigencias de las masculinidades hegemónicas. Las cuales se sustentan en las performances emocionales masculinas (Gosende, 2004), en este caso, negarse a hablar sobre la homosexualidad porque se es heterosexual.

En relación con el incidente asociado a la consigna de escritura, pero esta vez centrándonos en un problema de enseñanza de la lengua y la literatura, pensamos que puede estar operando una confusión de los roles autor, narrador, personaje. Ello lleva a inferir que los jóvenes consideran que si escriben en primera persona implica que su voz admite esa homosexualidad, una homosexualidad que en realidad es fruto de una interpretación de los lectores ya que en ningún momento en el texto literario se explicita. Es probable que el autor, Abelardo Castillo, al titular el cuento "El marica" juegue a condicionar la mirada. Asimismo, se podría pensar que busca problematizar los estereotipos de género ya que, como lectores, al advertir que César es "raro" (2007, p.47) y luego se niega a debutar con una prostituta, la reacción más generalizada, es interpretar que el personaje es homosexual. Esta confusión entre autor, narrador y personaje se encuentra enfatizada en el hecho de que el personaje principal -que es a la vez el narrador protagonista- se llama Abelardo, homónimo del autor. Esto puede advertirse en la respuesta de Facundo, quien considera que la identidad entre el narrador, el personaje y el autor es una manera de disfrazar la propia homosexualidad del autor. Como advertimos en el registro, la primera reacción es una negación total a realizar la consigna de escritura solicitada. Sin embargo, poco a poco podemos advertir que los estudiantes están "escribiendo" en su cabeza una posible carta. Es decir, al 
momento de preguntar a la docente qué es lo que deberían responder en ella y cómo deberían hacerlo se podría pensar que se están gestando posibles cartas en el plano imaginario que luego no son trasladadas al papel. Esta situación despierta algunos interrogantes relacionados con las concepciones históricas de la escritura como valor de documento y de permanencia.

Por otro lado, llama la atención cómo al momento de referirse al acto sexual entre la prostituta y los personajes, los estudiantes utilizan sin escrúpulos la palabra garchar en sus escritos, mientras que utilizan palabras y construcciones como "gay", "homosexual", "como él", haciendo alusión a la homosexualidad por omisión del término. Estas palabras no habían aparecido durante el debate oral, sino que los jóvenes se limitaron a la acepción de puto, hecho que despierta ciertos interrogantes en torno a las dimensiones de esta palabra y, nuevamente, la cuestión de dejarla o no asentada en el papel, en la escritura, en el documento.

Por último, nos resulta interesante advertir el uso de la parodia que realizan los estudiantes, y, principalmente, la avidez que detentan al realizar modificaciones "sobre la marcha" a medida que se está leyendo el texto por vez primera y en voz alta. Dubin (2016) se pregunta acerca de la existencia de una dualidad entre ciertos modos y usos de una cultura oficial y modos y usos de una cultura no oficial. Dualidad que relaciona con las distinciones que Rockwell establece entre historia documentada y no documentada (Rockwell y Ezpeleta, 1983; Rockwell, 2009), donde la historia no documentada es testimonio de la cotidianidad, de los sujetos sociales concretos que comparten el aula y las prácticas docentes efectivas. A partir de estas nociones, Dubin establece que "las lecturas paródicas dan cuenta de parte de esa historia no documentada. Una cotidianidad escolar que muestra lecturas literarias donde el cuerpo, el humor, la parodia, ocupan un lugar predominante" (2016, p. 84). Damos cuenta de esta operación paródica en el hecho de suplantar los personajes que aparecen en el relato, por personas que forman parte de la vida cotidiana de los jóvenes, conocidos por todos y, por ende, parte de un código de grupo. Código que se eligió ampliar - e incluir en él - a la docente al momento de aclarar que "la gordi" era una trabajadora social. Entendemos entonces que el uso de la parodia no tiene como finalidad burlar al otro, sino burlar al género en sí mismo, al relato legitimado. Esto puede advertirse en el hecho de que el estudiante que lee, se incluye él mismo en el juego paródico al intercambiar "el negro" por "yo". Siguiendo a Dubin:

La parodia, por tanto, funciona, principalmente, como un desplazamiento de las normas estéticas hegemónicas y la irrupción de una lectura subalterna; el humor operaría como un recurso que borra las jerarquías pero, también, como una concepción del mundo desacralizada, no solemne, que relativiza las verdades eternas, inmutables y únicas (2016, p. 88).

Por consiguiente, consideramos que el humor y la parodia por parte de los jóvenes podrían estar operando como una resistencia a las normas y cánones establecidos.

\section{CONCLUSIONES}

Sabemos que lxs jóvenes, independientemente de su contexto, clase social e inscripción sexo-genérica, tienden a buscar un grupo social de pertenencia. Pero el corrimiento de la norma, de lo esperado, en este caso el ser varones heterosexuales, no solo implicaría ser estigmatizado o ridiculizado sino que haría aún más difícil el tránsito por esta institución. Consideramos que, al interior de espacios carcelarios, la masculinidad, enfatizada en sus atributos de heterosexualidad y virilidad, se encuentra reforzada porque funciona como una herramienta de resistencia para sobrevivir la cotidianeidad del encierro.

Si bien el mandato social heterosexual es, prácticamente, algo incuestionable, gracias al presente registro podemos advertir que no por ello los estudiantes muestran algún tipo de rechazo o aversión que detente algo cercano a la homofobia sino, más bien, un cierto miedo a ser expulsado de la comunidad de pertenencia. Lopes Louro afirma que:

La inscripción de los géneros -femenino o masculino- en los cuerpos es hecha, siempre, en un contexto de una determinada cultura y, por lo tanto, con las marcas de esa cultura. Las posibilidades de la sexualidad -las formas de expresar los deseos 
y placeres- también son siempre socialmente establecidas y codificadas. Las identidades de género y sexuales son moldeadas por las redes de poder de una sociedad (1999, p. 2).

A su vez, las identidades sociales son definidas en el ámbito de la cultura y constituyen a los sujetos, ya que estos son interpelados a partir de diferentes situaciones, instituciones y agrupamientos sociales. Por ende, reconocerse en una identidad supone establecer un sentido de pertenencia a un grupo social de referencia.

Por último, consideramos que es de vital importancia contar con prácticas de enseñanza que hagan hincapié en las construcciones sociales de las sexualidades, que propicien situaciones áulicas de confianza y respeto donde lxs estudiantes y docentxs puedan manifestar sus opiniones, deseos e ideologías, que en algunas ocasiones pueden ir a contramano de lo esperado o motorizado por el contexto situacional, espacial y grupal.

Asimismo, se considera a la literatura, en todas sus formas, un vehículo que puede ayudar a deconstruir categorizaciones sexo-genéricas, a partir de la lectura de historias de otrxs, de focalizaciones distintas, que pueden abonar a la reflexión conjunta de distintos fenómenos. Al mismo tiempo, se cree en el valor de la escritura como posibilidad de posicionarse en otro lugar, de adoptar o ensayar otra voz, otro cuerpo.

En este sentido, nos permitimos pensar que, si como sostiene Butler (2017), las identidades no son fijas, estáticas, sino que son sucesivas escenificaciones, actos donde vamos resignificando una/s identidad/es de manera continua y constante, la literatura puede operar como ese espacio, con una cuota de ficción, donde los individuos se permitan adoptar distintos personajes y roles sexogenéricos, un espacio donde llevar adelante actos performativos de transformación, de ruptura, de metamorfosis. Actos que habiliten otras posibilidades. Actos subversivos.

\section{ReFERENCIAS}

Bergalli, R. (1980). La recaída en el delito: modos de reaccionar contra ella. Barcelona: Sertesa.

Bourgois, P. (2010). En busca de respeto. Vendiendo crack en Harlem. Buenos Aires: Siglo XXI.

Bonino, L (2004). Los micromachismos. Revista La Cibeles, 2.

Butler, J. (2017). El género en disputa. El feminismo y la subversión de la identidad. Buenos Aires: Paidós Studio.

Castillo, A. (2007 [1961]). "El marica” en Las otras puertas. Buenos Aires: Seix Barral.

Cesaroni, C. (2010). La vida como castigo: los casos de adolescentes condenados a prisión perpetua en Argentina. Buenos Aires: Grupo Editorial Norma.

Comité contra la tortura (2017). Informe Anual: El sistema de la Crueldad XI. La Plata, Comisión Provincial por la Memoria. Recuperado de: http://www.comisionporlamemoria.org/static/prensa/cct/informesanuales/Inform e_2017.pdf

Connell, R. (1987). Gender Regimes and the Gender Order. En Gender and Power (pp.119-14). Stanford: Stanford University Press.

Dubin, M. (2016). Parodias de alumnos al saber docente: literatura, cultura popular y humor. En Sawaya, $S$ y Cuesta, C (Coords.) Lectura y escritura como prácticas culturales. La investigación y sus contribuciones para la formación docente. (pp.83-89). Buenos Aires: Edulp.

García Borés, J. (2003). “El impacto carcelario” En Bergalli, R. Sistema penal y problemas sociales. España: Tirant lo Blanch.

Goffman, E. (2009). Internados: ensayos sobre la situación social de los enfermos mentales. Buenos Aires: Amorrortu.

Gosende, E. (2004). Accediendo al género masculino. Dimensiones históricas, hermenéutica, reflexiva y política de la masculinidad. Subjetividad y procesos cognitivos, 5, 159- 198.

Lagarde, M. (2001). Claves feministas para la negociación en el amor. Managua: Puntos de encuentro.

Lopes Louro, G. (1999). Pedagogia da sexualidade. En Lopes Louro, G (org.), O corpo educado. Pedagogias da sexualidade (pp. 9-42). Belo Horizonte: Ed. Autentica. 
Maduri, M. (2015). Sin berretines: Sociabilidad y movilidad intramuros. Una mirada etnográfica al interior de la prisión (Tesis de Licenciatura en Sociología). Universidad Nacional de San Martín, San Martín, Argentina.

Morgade, G. (1997). Mujeres en la educación: género y docencia en Argentina, 1870-1930. Buenos Aires: Miño y Davila.

Oleastro, I. (2018). ¿Qué nos dice un cuerpo? Cuerpo y sexualidades en la cárcel de varones. Cuestiones Criminales, 2(12), $1-28$.

Rockwell, E. (2009). La experiencia etnográfica. Buenos Aires: Paidós.

Rockwell, E.y Ezpeleta J. (1983) La escuela, relato de un proceso de construcción inconcluso. Ponencia presentada en Reunión de Clacso, Sao Paulo, Brasil.

Rubin, G. (1986). El tráfico de mujeres: notas sobre la economía política del sexo. En M. Navarro y C. Stimpson (Comps.). ¿Qué son los estudios de mujeres? México: FCE.

Rodríguez Alzueta, E. y Noya Garibaldi, N. (2016). Palabra y potencia: estrategias gramaticales contra la estigmatización. En Rodríguez Alzueta, E. (Comp.) Hacer bardo, provocaciones, resistencias y derivas de jóvenes urbanos. Buenos Aires: Malisia.

Valverde Molina, J. (1997). La cárcel y sus consecuencias. La intervención sobre la conducta desadaptada. Madrid: Ed. Popular.

Yannoulas, S. (1994). Educar: ¿una profesión de mujeres? La feminización del normalismo y la docencia (1870-1930). Buenos Aires: Kapelusz.

\section{Notas}

1 El Comité Contra la Tortura en su Informe Anual (2017) define estas prácticas como requisas vejatorias al cuerpo de las personas a través de prácticas violentas, inútiles y prolongadas, que suponen exposiciones al frío o al calor, inspecciones invasivas del cuerpo, imposición de posturas y/o ejercicios degradantes.

2 Los nombres de los estudiantes han sido modificados para resguardar sus identidades.

3 Maduri (2015) define los berretines como un concepto cultural propio del ámbito carcelario. La incorporación de berretines por parte de las personas privadas de su libertad supone la adquisición de determinados valores y prácticas inherentes al encierro que definirán su condición y movilidad dentro de la cárcel.

4 El engome es una práctica de aislamiento por castigo. Puede castigarse al todo el pabellón, implicando que no salgan del mismo o a personas en particular, impidiendo que estas salgan de sus celdas.

5 En el cuento se lee "verle la cara a Dios" como metáfora del acto sexual.

6 Las respuestas de los estudiantes han sido copiadas tal cual se encontraban en el manuscrito original.

7 Bondi: lío, quilombo.

8 Goffman caracteriza a las instituciones totales del siguiente modo: a) Todos los aspectos de la vida se desarrollan en el mismo lugar y bajo la misma autoridad. b) Todas las actividades se desarrollan junto con otros. c) Todas las actividades están estrictamente programadas. d) Todas las necesidades y todos los aspectos de la vida de los internos están sometidos a un plan predeterminado (2009, p. 21-22) 